\title{
Tooth supported overdenture- A preventive approach in prosthodontics
}

\author{
Varun Kumar ${ }^{1, *}$, Suyashvi Gupta ${ }^{2}$, Yogeshwari Krishnan ${ }^{3}$, Lakshya Vishnoi ${ }^{4}$ \\ ${ }^{1}$ Professor, ${ }^{2}$ PG Student, ${ }^{3}$ Lecturer, ${ }^{4}$ Intern, ${ }^{1,2}$ Dept. of Prosthodontics, Seema Dental College and Hospital, Rishikesh, \\ Uttarakhand, India \\ *Corresponding Author: Varun Kumar \\ Email: drvarun_smile@yahoo.co.in
}

\begin{abstract}
The purpose of maintaining the roots of one or more natural teeth in the oral cavity is to reduce the rate of ridge resorption, maintaining proprioception and providing alveolar bone support and achieve better stability of the denture with emphasis on psychological aspect of not being completely edentulous. This case report describes the utilization of the endodontically treated remaining natural roots to fabricate an overdenture that provided better retention than a conventional complete denture.
\end{abstract}

Keywords: Overdenture, Overlay denture, Proprioception, Post and core.

\section{Introduction}

Preventive prosthodontics emphasizes the importance of any procedure that can delay or eliminate future problems of form, function and esthetics. The goal of overdenture is to maintain teeth as a part of residual ridge. Instead of soft movable mucous membrane, the denture literally sits on teeth 'pilings', enabling the denture to withstand a much greater occlusal load without movement. The overdenture, a complete or partial denture prosthesis constructed over existing teeth or root structure, is not a new concept to solve a prosthodontic problem. Indeed, its use dates back over 100 years. Today, with the stress on preventive measures in prosthodontics, the use of overdentures has increased to the point where it is now a feasible alternative to most treatment outlines in the construction of prosthesis for patients with some remaining teeth. ${ }^{1}$ Overdenture is any removable dental prosthesis that covers and rests on one or more remaining natural teeth, the roots of natural teeth, and/or dental implants; a dental prosthesis that covers and is partially supported by natural teeth, natural tooth roots, and/or dental implants. ${ }^{2}$ Various terms have been used to describe this treatment modality: overlay denture, telescoped dentures, tooth supported dentures, hybrid prosthesis, crown and sleeve prosthesis, and the superimposing dentures. ${ }^{3}$

Over denture therapy is essentially a preventive prosthodontic concept since it attempts to conserve the few remaining natural teeth. There are two physiologic tenets related to this therapy: the first concerns the continued preservation of alveolar bone around the retained teeth ${ }^{4}$ while the second relates to the continuing presence of periodontal sensory mechanisms ${ }^{5}$ that guide and monitor gnathodynamic functions. Over dentures help to partly overcome many of the problems posed by conventional complete dentures like progressive bone loss, poor stability and retention, loss of periodontal proprioception, low masticatory efficiency, etc. ${ }^{6}$
The tooth must be treated endodontically to allow sufficient reduction of the clinical crown. Patients with single root teeth and only one canal that are easily negotiable are best candidates, although multirooted teeth can also be used. The present article discusses the case report of a patient with retained endodontically treated roots in maxillary and mandibuar arches and a full coverage crown on the maxillary canine. The treatment plan was to fabricate a tooth supported overdenture after doing post and core on the retained roots and the full coverage crown was retained as it is.

\section{Case Report}

A 62 year old female patient reported to the Department of Prosthodontics, Crown \& Bridge, Oral Implantology with the chief complaint of difficulty in chewing due to missing teeth and having desire to improve esthetics. On intraoral examination, endodontically treated roots with respect to $11,21,22$, 35 and 36, along with a full coverage crown on 23 were present [Fig. 1,2].

Patient was unwilling to remove the crown so a tooth supported overdenture was planned considering the feasibility and socio-economic status of the patient. Post and core was decided to be done on 11,21, 22 and 35.

After assessing endodontic therapy, canals of 11, 21, 22 and 35 were prepared for receiving cast metal post and core. Pattern resin (Pattern resin, GC corporation, Tokyo, Japan) was used to make patterns of post and core by direct technique [Fig. 3,4]. Patterns were casted using cast metal alloy and after that finishing and polishing were done. Metal copings were cemented on 11, 21, 22 and 35 [Fig. 5]. Also 36 was prepared to receive metal coping [Fig. 6].

Preliminary impression of maxillary and mandibular arches were then made with irreversible hydrocolloid (Zelgan2002 dust-free easy mixing, Dentsply India Pvt. Ltd., Haryana) [Fig. 7]. Impressions were poured in dental stone (Kalstone, Kalabhai Karson Pvt. Ltd., India) and custom tray was fabricated with 
self cure acrylic resin (Cold Cure Denture Base Material, Pyrax, Rapid Repair). Border molding was done with low fusing green stick compound (DPI Pinnacle) in both the arches [Fig. 8]. Dual impression was then made with zinc oxide eugenol impression material (DPI Impression Paste, Dental Products of India) and irreversible hydrocolloid (Zelgan2002 dustfree easy mixing, Dentsply India Pvt. Ltd., Haryana) was then made in over extended stock tray [Fig. 9]. Master casts were poured in dental stone (Kalstone, Kalabhai Karson Pvt. Ltd., India).

Maxillomandibular relationships were made [Fig. 10]. Master casts were mounted on the semi-adjustable articulator (Hanau Wide Vue Articulator). Shade selection was done for better esthetics. After doing teeth arrangement with artificial acrylic resin teeth, tryin was done [Fig. 11]. Dentures were processed in heat cure acrylic resin (Trevalon Denture Material, Dentsply India Pvt. Ltd., India). Dentures were finished, polished [Fig. 12,13,14] and inserted [Fig. 15,16].

Occlusion was evaluated and adjusted, Postoperative instructions were given and emphasis was given on instruction on adequate oral hygiene maintenance. After minor adjustments during post insertion visits, the patient was recalled.

Fig. 1,2: Intraoral view
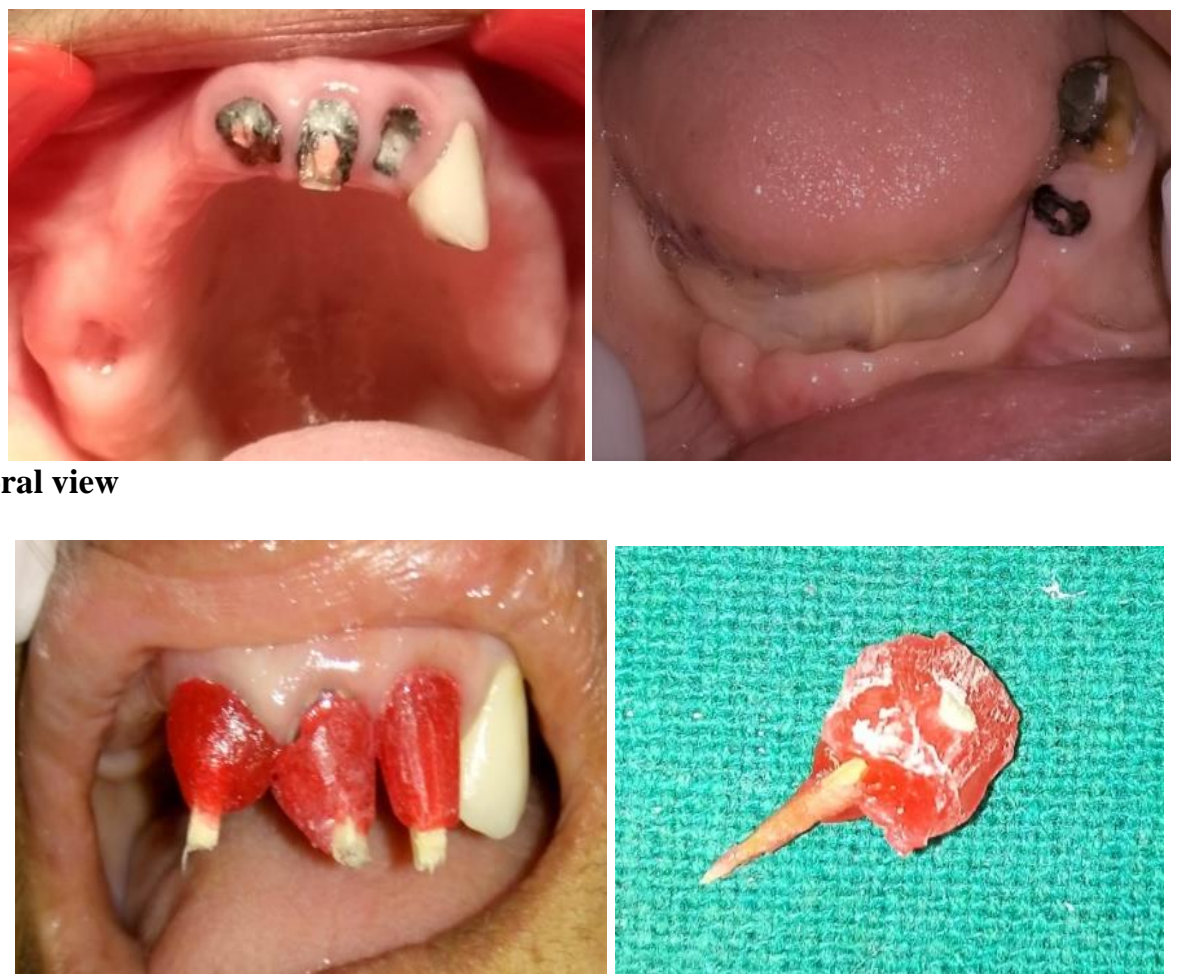

Fig. 3,4: Post and core
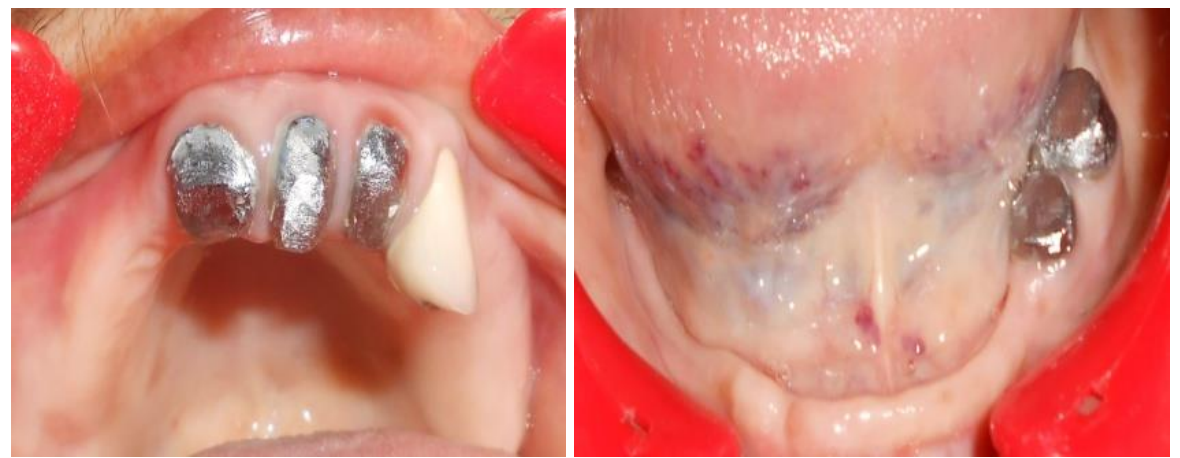

Fig. 5,6: Metal copings on the abutment teeth 


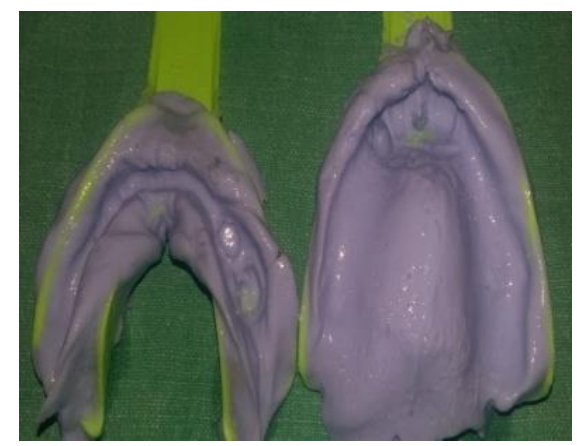

Fig.7: Primary impression

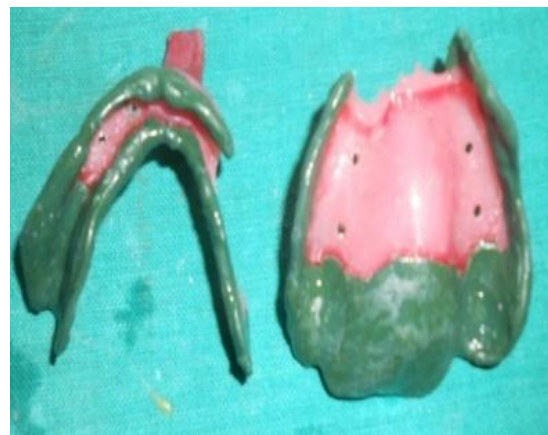

Fig. 8: Border molding

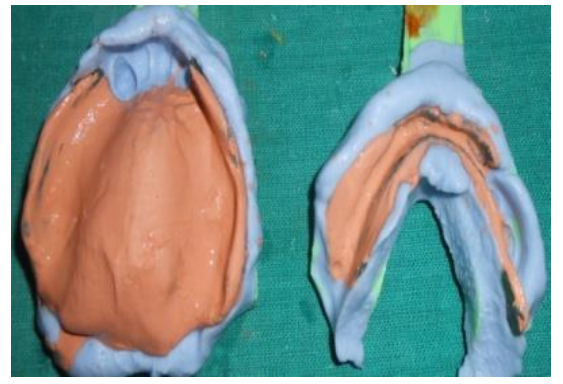

Fig. 9: Dual impression

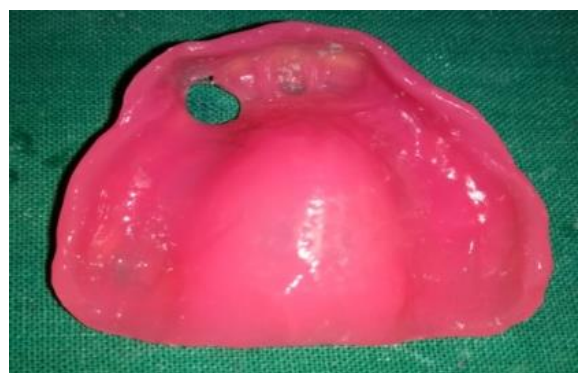

Fig. 13,14: Impression surface of denture
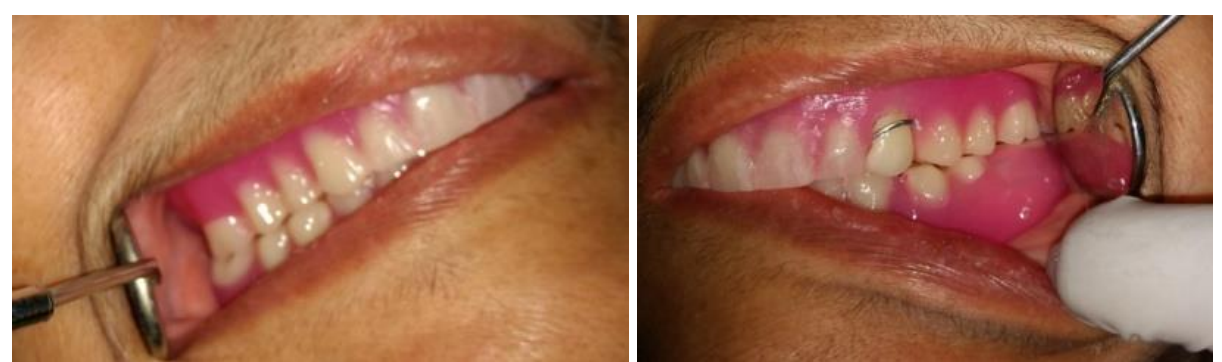

Fig. 10: Jaw relation

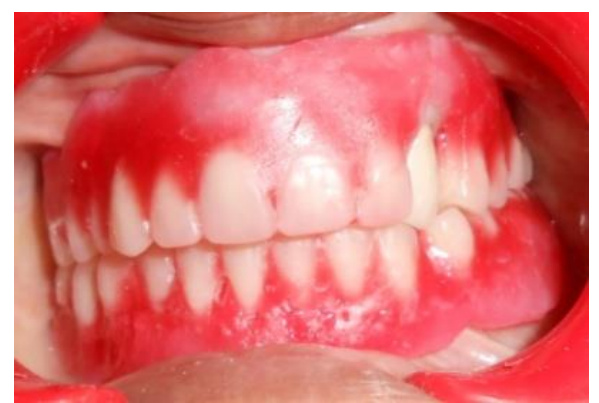

Fig. 11: Try-In

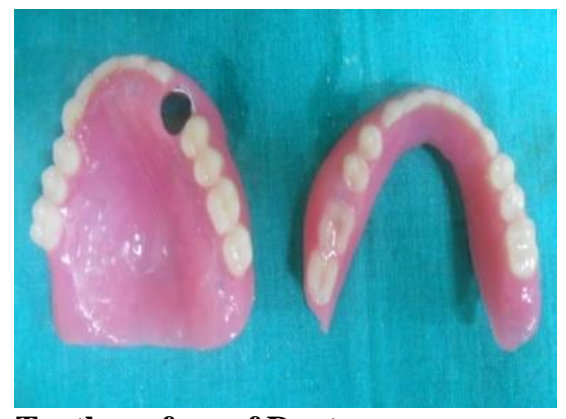

Fig. 12: Tooth surface of Denture

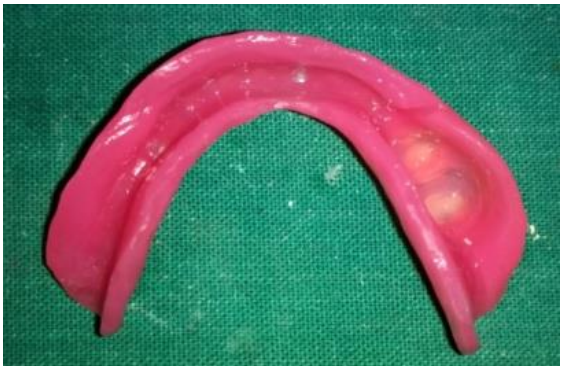

Fig. 15,16: Insertion 


\section{Discussion}

It is a documented fact that after the loss of the teeth the residual alveolar ridge undergoes rapid loss in all dimensions. The phenomenon of residual ridge resorption (RRR) following removal of teeth has been well observed and documented in literature. ${ }^{7,8}$

While the bone loss following the removal of teeth is stated to be rapid, progressive, irreversible and inevitable, it is equally well observed that bone is maintained around standing teeth and implants. ${ }^{8,9}$

Over denture therapy constitutes essentially a preventive prosthodontics concept as it endeavours to preserve the few remaining teeth and the supporting structures. The teeth which are too weak to support a fixed partial denture and are considered unsuitable to support a removable partial denture can often at times be usefully conserved and suitably modified to act as abutments under over dentures for useful span of time. ${ }^{10}$

As the over denture status of the prosthesis and its benefits to the patient depend solely on the continued retention of the underlying abutments, it becomes obligatory to periodically monitor their health and provide necessary steps to prolong their useful span. Here in lies the importance of periodical recall and review and patient motivation which makes over denture therapy a continued service.

This case report described the utilization of remaining roots in the oral cavity by doing post and core and fabricating an overdenture prosthesis over the metal copings. Tooth supported overdenture has many benefits over the conventional complete denture as it has improved retention. There is reduced rate of resorption and presence of proprioception provides better prognosis.

\section{Conclusion}

Tooth-supported overdentures may be considered as a good alternative to the conventional removable dentures, because they provide better retention, stability, support, stable occlusion, decrease in the forward sliding of the prosthesis and better control of the mandibular movements because of the proprioception feedback which increases the chewing efficiency and even phonetics, as compared to the conventional complete dentures. In present case report, the rate of the residual ridge resorption was decreased because of the transfer of compressive forces into the tensile forces by the periodontal ligament and better stress distribution.

\section{References}

1. Winkler S. Essentials of complete denture prosthodontics. 2013;505-531.

2. Glossary of Prosthodontic Terms. J Prosthet Dent. 2017;117(15):65.

3. Dhir RC. Clinical assessment of the Overdentures therapy. J Ind Prosthodont Soc. 2009;5:187-92.

4. Prince IB. Conservation of the supporting mechanism. $J$ Prosthet Dent. 1965;15:327-5.

5. Yalisove IL. Crown and sleeve coping retainers for removable partial prosthesis. J Prosthet Dent. 1966;16:1069-85.

6. Reitz PV, Weiner MG, Levin B. An overdenture survey: Preliminary report. J Prosthet Dent. 1977;37:246-58.

7. Toolson LB, Smith DE. A two year longitudinal study of overdenture patients, Part 1: Incidence and control of caries on overdenture abutments. J Prosthet Dent. 1978;40:486-91.

8. Tallgren A. Positional changes of complete dentures-A seven year longitudnal study. Acta Odontol Scand. 1969;27:539-61

9. Tallgren A. Positional changes of complete dentures-A seven year longitudnal study. Acta Odontol Scand. 1969;27:539-61.

10. Kumar V, Kalra P, Yunus N, Kalra S. Overdenture: A Boom for Tooth Supported Prosthesis. Heal Talk. 2011;4(1):26-27. 